\title{
Carbohydrate Hydrogel Products Do Not Improve Performance or Gastrointestinal Distress During Moderate-Intensity Endurance Exercise
}

\author{
Andy J. King \\ Australian Catholic University University of Leeds \\ Louise M. Burke \\ Australian Catholic University and \\ Australian Institute of Sport
}

\begin{abstract}
The benefits of ingesting exogenous carbohydrate (CHO) during prolonged exercise performance are well established. A recent food technology innovation has seen sodium alginate and pectin included in solutions of multiple transportable $\mathrm{CHO}$, to encapsulate them at $\mathrm{pH}$ levels found in the stomach. Marketing claims include enhanced gastric emptying and delivery of CHO to the muscle with less gastrointestinal distress, leading to better sports performance. Emerging literature around such claims was identified by searching electronic databases; inclusion criteria were randomized controlled trials investigating metabolic and/or exercise performance parameters during endurance exercise $>1 \mathrm{hr}$, with $\mathrm{CHO}$ hydrogels versus traditional CHO fluids and/or noncaloric hydrogels. Limitations associated with the heterogeneity of exercise protocols and control comparisons are noted. To date, improvements in exercise performance/capacity have not been clearly demonstrated with ingestion of CHO hydrogels above traditional $\mathrm{CHO}$ fluids. Studies utilizing isotopic tracers demonstrate similar rates of exogenous $\mathrm{CHO}$ oxidation, and subjective ratings of gastrointestinal distress do not appear to be different. Overall, data do not support any metabolic or performance advantages to exogenous $\mathrm{CHO}$ delivery in hydrogel form over traditional $\mathrm{CHO}$ preparations; although, one study demonstrates a possible glycogen sparing effect. The authors note that the current literature has largely failed to investigate the conditions under which maximal $\mathrm{CHO}$ availability is needed; high-performance athletes undertaking prolonged events at high relative and absolute exercise intensities. Although investigations are needed to better target the testimonials provided about $\mathrm{CHO}$ hydrogels, current evidence suggests that they are similar in outcome and a benefit to traditional $\mathrm{CHO}$ sources.
\end{abstract}

Keywords: encapsulated carbohydrate, glycogen, gut, sports nutrition, oxidation, sports drink

Recent interest in the 2-hr marathon (Caesar, 2019) has focused attention on an important sports nutrition strategy; consumption of carbohydrate $(\mathrm{CHO})$ during exercise to contribute to the substantial fuel costs of some endurance events. Events of sufficient intensity and duration to be limited by $\mathrm{CHO}$ availability benefit from an exogenous CHO supply (Stellingwerff \& Cox, 2014), with mechanisms including fuel provision once muscle glycogen is depleted (Coyle et al., 1986), spared liver (Gonzalez

(C) 2020 The Authors. Published by Human Kinetics, Inc. This is an Open Access article distributed under the terms of the Creative Commons Attribution-NonCommercial 4.0 International License, CC BY-NC 4.0, which permits the copy and redistribution in any medium or format, provided it is not used for commercial purposes, the original work is properly cited, the new use includes a link to the license, and any changes are indicated. See http://creativecommons.org/licenses/by-nc/4.0. This license does not cover any third-party material that may appear with permission in the article. For commercial use, permission should be requested from Human Kinetics, Inc., through the Copyright Clearance Center (http://www.copyright.com).

King and Burke are with the Mary Mackillop Institute for Health Research, Australian Catholic University, Melbourne, VIC, Australia. Burke is also with the Australian Institute of Sport, Canberra, ACT, Australia. Rowe is with the University of Leeds, Leeds, United Kingdom. King (andy.king@acu.edu.au) is corresponding author. et al., 2015; Wallis et al., 2006) and muscle (King et al., 2018; Tsintzas et al., 1995, 1996) glycogen use, and central nervous system benefits (Burke \& Maughan, 2015). A sliding scale of intake is recommended, according to event fuel needs and specific mechanisms underpinning performance benefits (Thomas et al., 2016). Upper targets for fuel-demanding events $\left(80-90+\mathrm{g} \cdot \mathrm{hr}^{-1}\right.$ $\mathrm{CHO}$ ), which aim to maximize the contribution of exogenous CHO to substrate use, are often challenged by the ability to consume, tolerate, and absorb large amounts of $\mathrm{CHO}$ (de Oliveira \& Burini, 2014). Factors include the availability of foods/drinks to meet $\mathrm{CHO}$ targets in practical amounts/volumes, the effect of the mode and intensity of exercise on gastrointestinal (GI) comfort and function (de Oliveira \& Burini, 2009), the role of specific "gut training" (Cox et al., 2010), and characteristics of the $\mathrm{CHO}$ source. Here it has been shown that the use of CHO blends ("multiple transportable CHO" such as glucose [G] and fructose [F]) can maximize gut uptake via the use of different intestinal transport mechanisms, assisting with substrate delivery and the management of gut comfort (Jeukendrup, 2010).

Recently, specialized sports foods claiming to address such factors via the use of "hydrogel technology" have become commercially available (Sutehall et al., 2018). These supplements, combining typical $\mathrm{CHO}$ sources with pectin (a soluble fiber) 


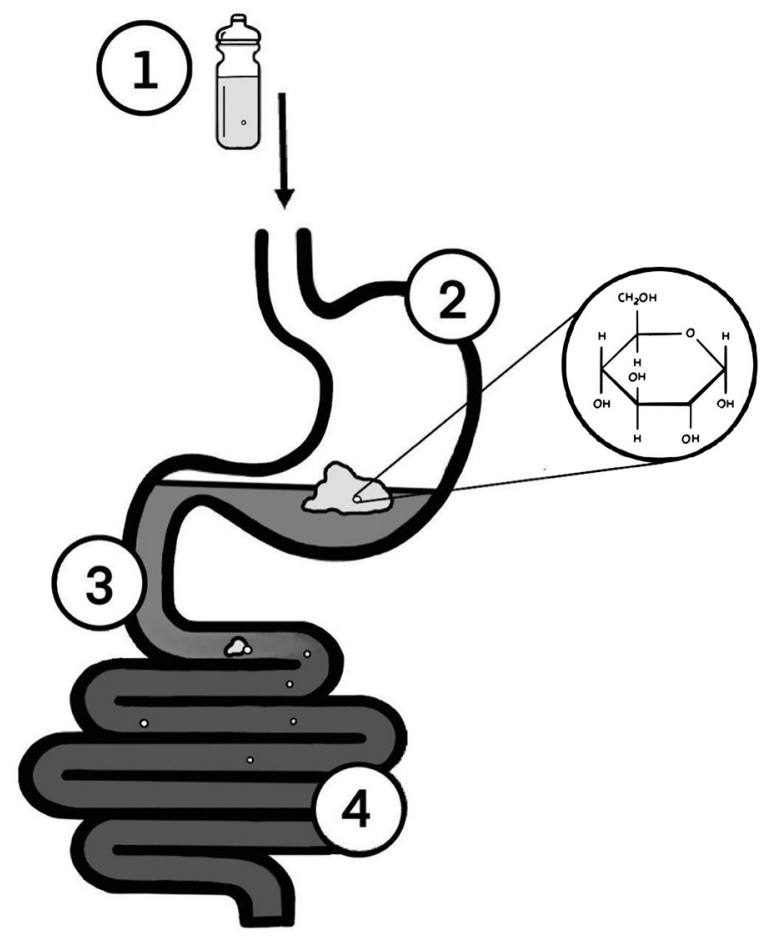

1

Gelling agents such as alginate and pectin are added to typical $\mathrm{CHO}$ (e.g., glucose, fructose, sucrose) in solution.

2

On contact with gastric fluid, $\mathrm{CHO}$-alginate mixtures form a hydrogel, encapsulating the $\mathrm{CHO}$ in the solution.

3

The encapsulated $\mathrm{CHO}$ passes into the duodenum, without activating glucagon-like peptide 1 secretion in the enteroendocrine cells of the gastrointestinal mucosa. Gastric emptying is therefore not reduced as with typical high $\mathrm{CHO}$ drinks.

In the duodenum, the higher $\mathrm{pH}$ environment dissolves the hydrogel allowing intestinal $\mathrm{CHO}$ transport proteins to deliver the ingested $\mathrm{CHO}$ to the systemic circulation.

Figure 1 - Mechanisms of CHO hydrogel formation and delivery to the small intestine. Despite benefits to gastric emptying with hydrogelencapsulated $\mathrm{CHO}$, the rate-limiting step of exogenous $\mathrm{CHO}$ oxidation lies in the intestinal transport of monosaccharides. $\mathrm{CHO}=$ carbohydrate.

and alginate (a polymer derived from seaweed) undergo gelation on contact with low $\mathrm{pH}$ solutions, such as stomach acid, to encapsulate the CHO (Marciani et al., 2019). Enhanced rates of gastric emptying could deliver this "hydrogel" to the small intestine where it dissolves in the higher $\mathrm{pH}$ environment for absorption, leading to reduced gut discomfort, enhanced muscle CHO delivery, and performance benefits (Figure 1). Indeed, according to testimonials, the commercial product has been quickly adopted by elite athletes (Sutehall et al., 2018) and publicized in sporting successes including the 1:59 marathon project, leading to marketing claims that it is the "world's fastest sports fuel" (Maurten, 2020). Noting that this has largely occurred in the absence of scientific validation of these claims, we undertook a review of newly published investigations of hydrogel CHO to determine whether they achieve better GI characteristics, substrate delivery, and performance effects under exercise conditions than traditional sports drinks and gels.

\section{Methods}

A search of electronic databases for studies published up to May 14, 2020 was independently completed by two authors (A. King/ J. Rowe), with the key methodological process and considerations involved in including/excluding data summarized in Figure 2.

To be eligible for this review, studies were required to have investigated a $\mathrm{CHO}$ hydrogel compound during prolonged, endurance exercise defined as continuous running, cycling, triathlon, rowing, swimming, and cross-country skiing greater than 1-hr duration. Studies with exercise durations lasting $1 \mathrm{hr}$ or less were excluded because $\mathrm{CHO}$ ingestion is unlikely to be beneficial during shorter duration exercise (Burke et al., 2011; Thomas et al., 2016). CHO mouth rinse studies were also excluded as the primary mechanism whereby performance is improved is neurological in origin.
The CHO hydrogel used could be a commercially available product or a laboratory-manufactured solution, provided that the active substance included as a gelling agent was known to encapsulate ingested $\mathrm{CHO}$ in the stomach. A control comparison/ condition was required to be a typical $\mathrm{CHO}$ control, matched for $\mathrm{CHO}$ dose and type, or a placebo. Studies were included if they reported data on one or more of physiological or performance variables (Table 1). Review articles and case studies were excluded.

From the available studies, between-condition differences for hydrogel and comparison products were calculated. Standardized effect sizes (ES) were calculated using Hedge's $g$ adjustment for small samples with $95 \%$ confidence intervals for the ES computed. No statistical adjustments for ES were made for carryover effects, since suitable washout periods were included in these crossover trials. No assessment of publication bias was undertaken with the low number of studies and the likely presence of heterogeneity; we noted that this can affect the robustness of publication bias analysis (Ioannidis \& Trikalinos, 2007). Forest plots were produced to provide a visual comparison of effects in studies measuring exercise performance.

\section{Results}

We located six studies (Table 1) which compared a CHO hydrogel containing alginate and/or other gelling compounds with a noncaloric hydrogel placebo $(n=1)$ or $\mathrm{CHO}$ fluids of matched $\mathrm{CHO}$ $(n=5)$. Although $\mathrm{CHO}$ hydrogels were similar in composition, containing a mixture of maltodextrin (MD) and F, total $\mathrm{CHO}$ ranged from 68 to $132 \mathrm{~g} \cdot \mathrm{hr}^{-1}$. Five of the six studies included a matched condition for dose and type of $\mathrm{CHO}$ (i.e., $\mathrm{MD}+\mathrm{F}$ fluid) and two studies included a G or MD only fluid matched for $\mathrm{CHO}$ dose. Therefore, four studies met guidelines for upper range $\mathrm{CHO}$ 


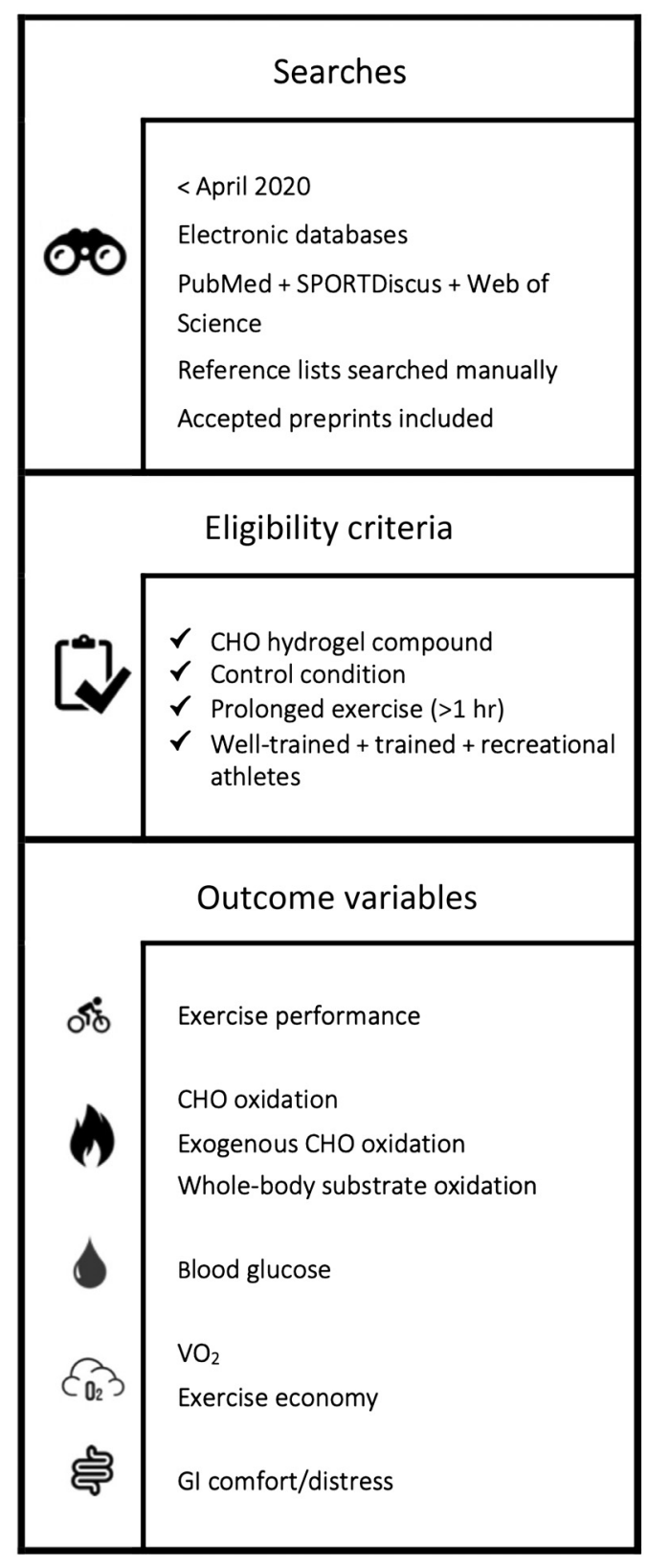

Figure 2 - Methodology and review considerations. GI = gastrointestinal; $\mathrm{CHO}=$ carbohydrate.

intakes: $\sim 90 \mathrm{~g} \cdot \mathrm{hr}^{-1}$ from multiple transportable $\mathrm{CHO}$ sources (Jeukendrup, 2010).

All studies were randomized controlled crossover trials. They investigated trained male athletes $\left(\mathrm{VO}_{2} \max 55-70 \mathrm{ml} \cdot \mathrm{kg}^{-1} \cdot \mathrm{min}^{-1}\right)$, with the exception of Pettersson et al. (2019) and (Flood et al., 2020), who included six female cross-country skiers $\left(\mathrm{VO}_{2} \max\right.$ : $\left.59.9 \pm 2.6 \mathrm{ml} \cdot \mathrm{kg}^{-1} \cdot \mathrm{min}^{-1}\right)$ and seven female cyclists $(54.3 \pm$ $12.3 \mathrm{ml} \cdot \mathrm{kg}^{-1} \cdot \mathrm{min}^{-1}$ ). Exercise modes included cycling (four studies), running (one), and cross-country skiing (one). A period of fixed-intensity exercise was included in all protocols (90-180 min) with five of the six studies then measuring exercise performance/ capacity using a time trial (three studies), time to exhaustion test (one), or repeated sprint test (one). Each of the studies in which performance was measured involved a preexercise meal according to current recommendations (Burke et al., 2011) or a self-selected
"CHO-rich" meal (Flood et al., 2020). The only study undertaken under fasting conditions (Barber et al., 2020) focused on exogenous $\mathrm{CHO}$ oxidation as the primary outcome, which is not affected by prior muscle glycogen content (Margolis et al., 2019). Betweenconditions comparisons remain valid despite the difference in breakfast protocol to other studies.

\section{Exercise Performance}

Performance was similar with $\mathrm{MD}+\mathrm{F}$ hydrogel, isocaloric $\mathrm{MD}+\mathrm{F}$ fluids (Figure 3; Baur et al., 2019; Flood et al., 2020; McCubbin et al., 2019; Mears et al., 2020b), or noncaloric hydrogel (Pettersson et al., 2019). Relative performance changes between $\mathrm{CHO}$ hydrogel and fluids ranged between $+1.05 \%$ and $+3.8 \%$ but were not statistically significant $(p<.05)$. The largest change reported by Mears et al. (2020b) during a 20-min fixed-work time trial was a moderately higher workload (3.8\% improvement, $E S=0.51)$ with $\mathrm{CHO}$ hydrogel ingestion compared with the dose matched $\mathrm{MD}+\mathrm{F}$ control. All other performance effects were very small $(E S<0.10)$.

\section{Physiological Measures}

Exogenous $\mathrm{CHO}$ oxidation was measured in two studies, but only one included a comparative $\mathrm{CHO}$ condition (Barber et al., 2020) with exogenous $\mathrm{CHO}$ oxidation peaking at $1.1 \pm 0.3 \mathrm{~g} \cdot \mathrm{min}^{-1}$ in both the $\mathrm{MD}+\mathrm{F}$ hydrogel and MD + F fluid (Table 1$) . \mathrm{MD}+\mathrm{G}$ ingestion resulted in a moderately lower oxidation rate $\left(0.92 \pm 0.3 \mathrm{~g} \cdot \mathrm{min}^{-1}\right)$. Total exogenous $\mathrm{CHO}$ oxidation over the final (second) hour of running was not modified by $\mathrm{MD}+\mathrm{F}$ hydrogel $(48.25 \pm 16.5 \mathrm{~g}$ vs. $50.25 \pm 16.5 \mathrm{~g}$ in $\mathrm{MD}+\mathrm{F}$ solution) but both were higher than $\mathrm{MD}+\mathrm{G}(41.25 \pm 15.0 \mathrm{~g})$. Pettersson et al. (2019) reported exogenous $\mathrm{CHO}$ oxidation of $1.22(0.89-1.66) \mathrm{g} \cdot \mathrm{min}^{-1}$ with $\mathrm{MD}+\mathrm{F}$ hydrogel, indicating that a higher CHO dose (132 g.hr $\left.{ }^{-1}\right)$ with hydrogel may result in higher exogenous $\mathrm{CHO}$ utilization.

Total rates of $\mathrm{CHO}$ and fat oxidation during the steady-state exercise varied between studies, a consequence of different exercise intensities. Therefore, it is of interest to examine relative contributions to total fuel use (Figure 4) as well as absolute rates of oxidation (Table 1). When comparing MD + F in hydrogel versus fluid form, divergent effects were reported. Mears et al. (2020b) and Baur et al. (2019) found no differences in relative substrate contribution during submaximal cycling at $50 \% \mathrm{~W}_{\max }$ but the nonsignificant increase in fat oxidation (and decrease in $\mathrm{CHO}$ oxidation) reported by Baur et al. (2019) is notable, and consistent with Barber et al. (2020). Flood et al. (2020) also reported similar CHO and fat oxidation with $\mathrm{CHO}$ and hydrogel ingestion during low-intensity cycling. However, Baur et al. (2019) also noted lower absolute total CHO and fat (to Mears et al. [2020b] in both conditions), despite similar ingestion rates (68 and 78 g.hr ${ }^{-1}$ ). McCubbin et al. (2019) did not report differences in substrate oxidation during steady-state running, and neither $\mathrm{CHO}$ nor fat oxidation were modified by the hydrogel form during a subsequent incremental test. Barber et al. (2020) however, reported a reduced contribution of endogenous $\mathrm{CHO}$ during steadystate running with hydrogel $\mathrm{CHO}$. Glycogen contributed $\sim 60 \%$ of total energy expenditure with $\mathrm{MD}+\mathrm{G}$ and $\mathrm{MD}+\mathrm{F}$ fluids, but $\sim 50 \%$ with MD+ F hydrogel. Differences in glycogen use were explained by higher exogenous $\mathrm{CHO}$ oxidation in comparison with $\mathrm{MD}+\mathrm{G}$ fluid, and increased fat oxidation in relation to $\mathrm{MD}+\mathrm{F}$ fluid (Figure 4). Comparisons between studies employing different exercise modalities for whole-body substrate utilization are difficult as noted previously (Achten et al., 2003). 


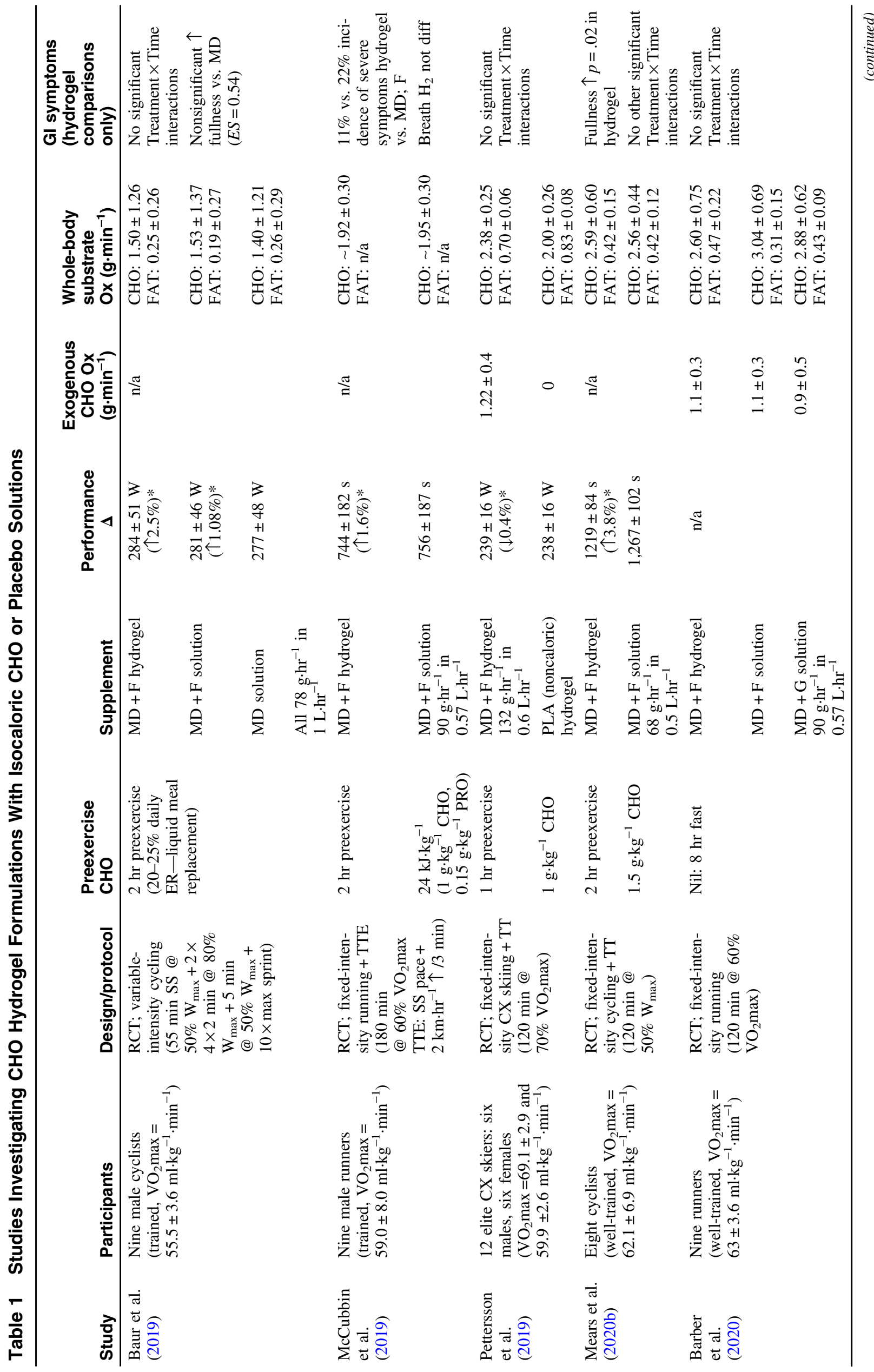




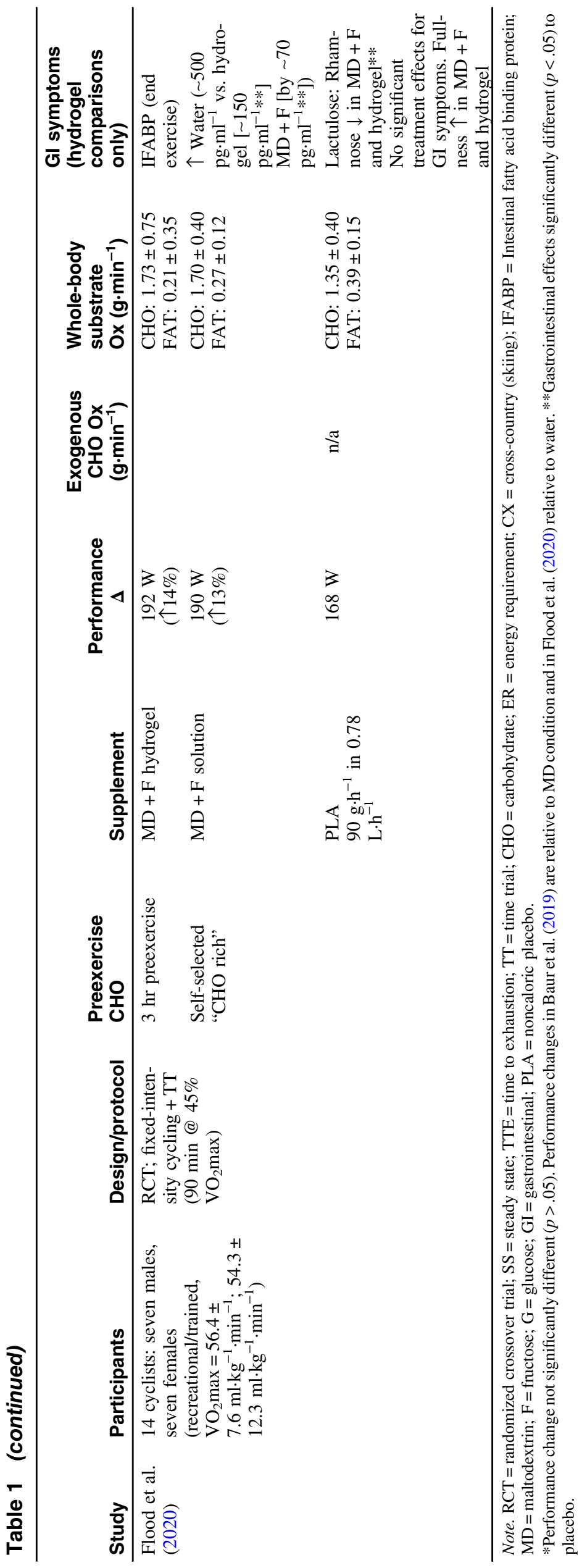

IJSNEM Vol. 30, No. 5, 2020 


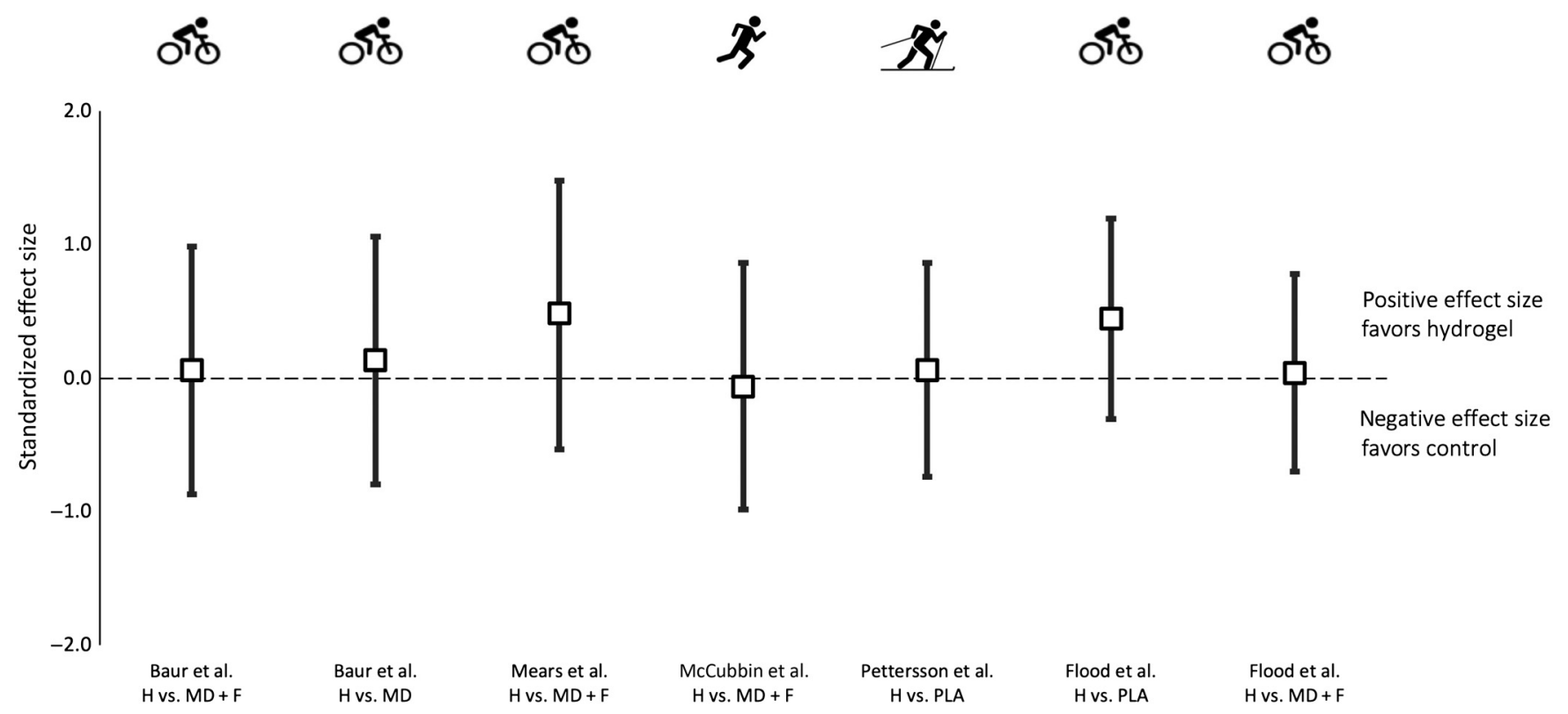

Figure 3 - Forest plot of standardized effects sizes and 95\% confidence intervals for exercise performance in studies comparing CHO hydrogel formulations with isocaloric $\mathrm{CHO}$ or noncaloric placebo solutions. $\mathrm{H}=$ hydrogel; $\mathrm{MD}=$ maltodextrin; $\mathrm{MD}+\mathrm{F}=$ maltodextrin + fructose; $\mathrm{PLA}=$ placebo; $\mathrm{H}(\mathrm{MD}+\mathrm{F})=$ hydrogel of $\mathrm{MD}+\mathrm{F} ; \mathrm{CHO}=$ carbohydrate.

Gastrointestinal discomfort was reported in all studies with similar responses from the hydrogel trial. McCubbin et al. (2019) reported a slightly, but not significantly, higher overall incidence of GI symptoms while running with $\mathrm{MD}+\mathrm{F}$ fluids than $\mathrm{MD}+\mathrm{F}$ hydrogel (22\% vs. 11\%). In cycling, Mears et al. (2020b) and Baur et al. (2019) reported slightly increased stomach fullness with $\mathrm{CHO}$ hydrogel ingestion but similarly, differences were not significant. Fullness in Flood et al. (2020) did not differ between CHO hydrogel and MD + F. Baur et al (2019) also reported moderately, but not significantly increased nausea with the hydrogel compared with MD + F fluids ( $E S=0.53, p=.23)$. Data from Pettersson et al. (2019) reported low levels of GI issues during skiing in cold conditions in either trial, but a small, nonsignificant decrease in stomach rumbling with the $\mathrm{MD}+\mathrm{F}$ hydrogel in comparison with a noncaloric hydrogel. In the only study to report GI data through nonsubjective measures, Flood et al. (2020) found significantly higher intestinal fatty acid-binding protein and lactulose to rhamnose ratio with placebo compared with both $\mathrm{CHO}$ hydrogel and matched $\mathrm{MD}+\mathrm{F}$ ingestion.

\section{Discussion}

This is a timely investigation of the evidence that intake of hydrogel encapsulations of multiple transportable $\mathrm{CHO}$ during prolonged endurance exercise provides benefits over traditional sports drinks, in response to the recent interest in newly available commercial hydrogel products. We summarized studies where hydrogels, formed by combining $\mathrm{MD}+\mathrm{F}$ with gelling agents such as alginate and pectin, were compared with typical $\mathrm{CHO}$ fluids containing single or multiple $\mathrm{CHO}$ sources, or a noncaloric hydrogel treatment. Despite marketing claims and lay media discussion about $\mathrm{MD}+\mathrm{F}$ products in hydrogel format, currently available studies fail to show benefits in terms of muscle oxidation of exogenous $\mathrm{CHO}$, GI comfort, or performance. The current literature, comprised of robust randomized controlled trials, is small and includes nuances around total substrate oxidation and gut comfort due to exercise mode and intensity, as well as total CHO intake. Furthermore, the conditions under which it is promoted to achieve its key benefits (high rates of $\mathrm{CHO}$ intake during prolonged exercise at high absolute and relative intensities) have not been investigated, potentially due to the challenge of involving elite competitors within traditional research protocols and the technical challenges of undertaking measurements of interest (e.g., gastric emptying, tracer determined substrate oxidation) under these conditions. While further studies with relevant protocols are needed to investigate the putative benefits of these products, the present literature fails to endorse the marketing claims.

Over the past 5 years, CHO-containing drinks that achieve hydrogel encapsulation within the gut have become commercially available, with claims that they achieve superior $\mathrm{CHO}$ delivery to the muscle for lower GI distress, leading to performance benefits over traditional $\mathrm{CHO}$-containing sports products (Maurten, 2020; Sutehall et al., 2018). CHO hydrogel products have attempted to improve on existing nutrition recommendations (use of CHO with multiple transporters [Jeukendrup, 2010] and gut training [Jeukendrup, 2017]) to target the need for highperformance athletes to achieve high $\mathrm{CHO}$ availability during prolonged events conducted at high relative and absolute intensities (Burke et al., 2019). Although there is evidence that these products achieve gelation within the acidic stomach environment as claimed (Marciani et al., 2019; McCubbin et al., 2019), subsequent effects on gastric emptying, intestinal absorption, and delivery to the muscle during exercise remain largely untested. Our review summarized the available literature on the use of these products during prolonged exercise in terms of GI comfort, substrate utilization, and performance. The scarcity and heterogeneity of study protocols prevents a metaanalytical approach from producing meaningful results. However, in view of community interest and marketing claims, we felt it was timely to collate the findings of available studies in narrative form to summarize the overall findings and alert researchers to the need for particular protocols. 
The current literature involves studies of subelite athletes (although one study of elite performers is noted [Pettersson et al., 2019]), exercising over 2-3 hr at intensities of approximately 45-70\% $\mathrm{VO}_{2}$ peak. Such protocols likely represent pragmatic choices based on subject availability and the technical requirements around the steady-state conditions needed to monitor muscle substrate use during exercise (Robert et al., 1987). According to our review, although the GI handling of these products has not been directly measured, these studies have failed to find evidence of increased oxidation of exogenous $\mathrm{CHO}$ when $\mathrm{MD}+\mathrm{F}$ is provided in hydrogel form compared with conventional solutions. Indeed, rates of exogenous $\mathrm{CHO}$ oxidation derived from ${ }^{13} \mathrm{C}$ isotopic tracer techniques are in line with results of previous studies of traditional CHO-containing fluids (King et al., 2019; O'Brien et al., 2013), or in the case of studies in which direct comparison has been made between $\mathrm{CHO}-$ matched fluids and hydrogel products, no differences in exogenous $\mathrm{CHO}$ use has been detected (Barber et al., 2020). Since the rate-limiting step of exogenous CHO oxidation is believed to lie in the GI tract and not cellular glucose uptake (Hawley et al., 1994), this indirectly indicates that CHO hydrogels have not achieved a net benefit to the gastric emptying/intestinal $\mathrm{CHO}$ transport processes.

The studies published to date confirm previous knowledge that whole-body $\mathrm{CHO}$ utilization is altered by the intake of $\mathrm{CHO}$ during exercise (Pettersson et al., 2019) and by the choice of multiple transportable $\mathrm{CHO}$ sources that increase the capacity for total intestinal absorption (Barber et al., 2020; Baur et al., 2019). In general, Figure 4 suggests that hydrogel encapsulation per se does not change total $\mathrm{CHO}$ oxidation during exercise (Baur et al., 2019; Flood et al., 2020; McCubbin et al., 2019; Mears et al., 2020b).
However, Barber et al. (2020) reported a decrease in endogenous $\mathrm{CHO}$ utilization and increase in fat oxidation when high rates of $\mathrm{CHO}$ intake were consumed in hydrogel form versus fluid. Muscle glycogen sparing effects have been reported in several studies of $\mathrm{CHO}$ intake during endurance exercise, but performance improvements have not been consistently observed (Newell et al., 2014). Previous work from our group (King et al., 2018) and others (Smith et al., 2010) has shown that the muscle glycogen response to $\mathrm{CHO}$ ingestion is dose dependent. Precise mechanisms to explain this effect have not been investigated, but likely sit within the cellular flux through the glycolytic pathway and the interaction of exogenous glucose and glucosyl units liberated from glycogen at glucose-6-phosphate. Exogenous $\mathrm{CHO}$ more consistently reduces liver glycogen use as long as the ingested dose is sufficient to inhibit hepatic glycogenolysis and glucose output (Gonzalez \& Betts, 2019). However, liver glycogen capacity is much smaller than muscle ( $100 \mathrm{~g}$ vs. $400-500 \mathrm{~g}$ ) and complete liver glycogen sparing during exercise has only been reported with an extremely high CHO dose (Jeukendrup et al., 1999). The ingested doses in the reviewed studies cover a wide range, and only one study estimated whole-body endogenous glycogen utilization using expired ${ }^{13} \mathrm{CO}_{2}$ tracer methods, which do not account for specific liver and muscle contributions; conclusions around a dose effect with $\mathrm{CHO}$ hydrogels are not possible at this stage. However, if an event requires maximized $\mathrm{CHO}$ availability, recommendations to saturate intestinal CHO transporters should remain if athletes tolerate these doses in terms of GI distress.

As has been the case in previous investigations of $\mathrm{CHO}$ feeding during exercise, methodological differences between studies do not allow firm interstudy comparisons. Factors such as $\mathrm{CHO}$

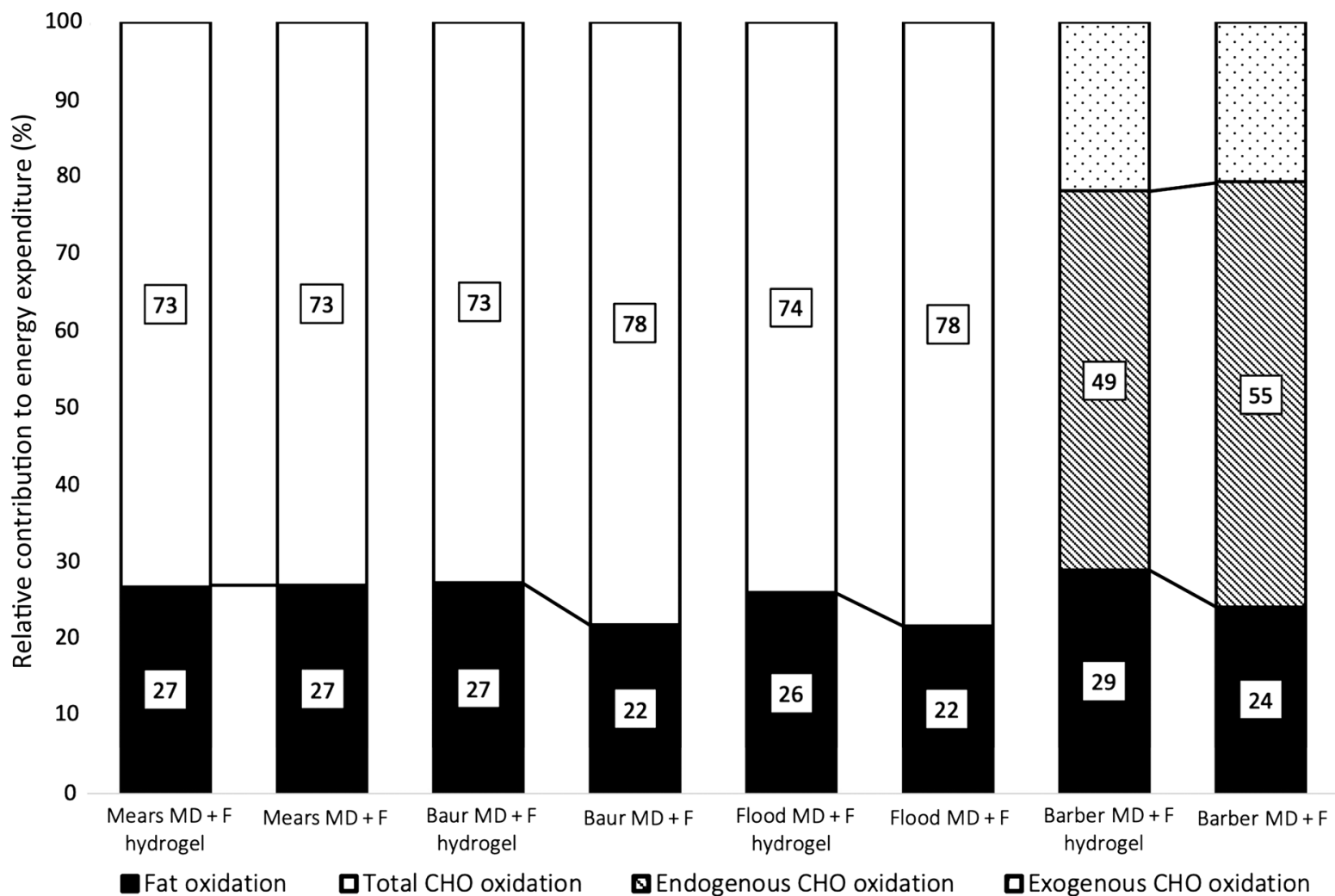

Figure 4 - Comparison of relative contributions to energy expenditure from fat (black bars) and carbohydrate (white bars) with maltodextrin and fructose $(\mathrm{MD}+\mathrm{F})$ ingestion in fluid and hydrogel form. Exogenous (dotted bars) and endogenous (hashed bars) contributions shown where data were available. $\mathrm{MD}+\mathrm{F}=$ maltodextrin + fructose $; \mathrm{CHO}=$ carbohydrate . 
dose, as well as timing, exercise intensity, mode and duration, prefeeding state, and potentially training status create differences in outcomes. Although such variability creates difficulty in piecing together an emerging literature, it is important to recognize that many of these factors may be fixed or characteristic of specific sporting events. Further studies, particularly those using multiple conditions within the same investigation, might help to isolate any conditions under which hydrogel $\mathrm{CHO}$ might provide an advantage. Exercise intensity has important implications for intestinal absorption due to the diversion of splanchnic blood flow at higher intensities (ter Steege \& Kolkman, 2012). Therefore, potential mechanistic benefits of $\mathrm{CHO}$ hydrogels in terms of GI tolerance and absorption may only be observed in the higher intensity domains, presenting an opportunity for future research. To date, only one study (Pettersson et al., 2019) has investigated what may be considered higher intensity exercise. Running also utilizes less localized muscle recruitment compared with cycling, resulting in increased surface area blood flow and a greater reduction of splanchnic blood flow (de Oliveira et al., 2014). Similar responses are likely in cross-country skiing given the whole-body nature of the sport and may even demand higher $\mathrm{CHO}$ utilization (Losnegard et al., 2014). While body mass is not considered a methodological factor for exogenous CHO oxidation (Jeukendrup, 2010), the combination of differences in exercise mode and intensity in the reviewed studies does not allow for firm extrapolations to higher intensity exercise, which may also benefit further from optimal, that is, at intestinal saturation, $\mathrm{CHO}$ dosing. Due to the increased duration of training and competition, cyclists may be at an advantage over runners if habituated to $\mathrm{CHO}$ intake. Increased $\mathrm{CHO}$ exposure causes a "gut training" effect (Cox et al., 2010), leading to enhanced GI tolerance of ingested $\mathrm{CHO}$ during exercise. Gut tolerance is therefore a further methodological factor that differentiates between exercise modality and may mean potential GI benefits to $\mathrm{CHO}$ hydrogels may be more likely in individuals with lower natural $\mathrm{CHO}$ tolerance or with less habitual $\mathrm{CHO}$ gut training. This would serve as a useful consideration or screening tool in future study design.

Typically, the intake of solutions with high $\mathrm{CHO}$ content during prolonged exercise delays gastric emptying and is associated with higher incidence of GI distress (Rehrer et al., 1992). A positive finding from this review is that $\mathrm{MD}+\mathrm{F}$ hydrogel formulations were generally well tolerated across the range of doses and exercise protocols that were examined. However, hydrogel solutions did not improve GI tolerance per se above comparable $\mathrm{CHO}$ sources in traditional fluid form. It remains to be seen if they systematically reduce GI symptoms at higher doses approaching and above intestinal saturation, due to specific interaction with the digestive system. Reports of a slight increase in gastric fullness associated with the hydrogel are of interest (Baur et al., 2019), since even if there is a subsequent increase in gastric emptying associated with the formation of the gel, it may create an initial sensation of fullness. Quantitative measures of GI barrier function and damage reported by Flood et al. (2020) evidence that CHO hydrogels do not provide a further protective effect to the intestinal membrane over typical $\mathrm{CHO}$ ingestion. These data do however, confirm the preventative role of $\mathrm{CHO}$ for enterocyte injury and small intestine permeability during endurance exercise (Snipe et al., 2017).

Although an enhancement of gastric emptying is the mechanism most heavily marketed in support of the use of the hydrogel sports drinks, the importance of gastric emptying in the whole process of delivering $\mathrm{CHO}$ from the mouth to the muscle mitochondria, and any benefits achieved by hydrogels, are difficult to ascertain. So far, the available evidence is limited to a report by
Sutehall et al. (2020) that a commercially available MD $+\mathrm{F}$ hydrogel increased gastric emptying compared with $\mathrm{G}+\mathrm{F}$ and $\mathrm{MD}+\mathrm{F}$ solutions when consumed as a bolus at rest, with time to empty half the drink being nearly twice as fast for MD + F hydrogel (21 $\mathrm{min})$ than its fluid counterpart (37 $\mathrm{min})$. Despite significantly less volume remaining in the stomach at $30 \mathrm{~min}$, differences became increasingly smaller thereafter (Sutehall et al., 2020). Since none of the currently available studies have attempted to directly measure gastric emptying during exercise, it is not possible to comment on what occurs under these circumstances. However, according to the general literature, gastric emptying is not considered to be the rate-limiting step in determining the availability of exogenous fuels consumed during exercise (see review by Rowlands et al. [2015]) and its measurement includes artifacts and practical difficulties. Furthermore, without using complex invasive measures (Shi et al., 1995), intestinal absorption cannot be directly measured; therefore, the endpoint of muscle $\mathrm{CHO}$ oxidation is used to reflect the contribution of a combination of gut processes between consumption and delivery to the mitochondria. The evidence collected from the currently available studies does not show clear evidence of differences in the overall process.

Even if hydrogel-encapsulated $\mathrm{CHO}$ can be shown to have different gut characteristics per se, the effects of the amount, timing, and pattern of intake of CHO sources on gastric emptying are among the many interacting factors that should be considered in the current story. This is of interest since in many sporting events, the pattern of intake is dictated by the availability of fluids at breaks or feed stations rather than continuous or spontaneous access. Recent work by Mears et al. (2020a) reported that mean and peak exogenous $\mathrm{CHO}$ oxidation is altered slightly (but significantly) by $\mathrm{CHO}$ timing, with better outcomes associated with consuming sources every $20 \mathrm{~min}$ compared with 5-min intervals. Meanwhile, Menzies et al. (2020) reported better endurance when $\mathrm{CHO}$ intake commenced early during a running protocol than later delivery. Therefore, future research should systematically investigate conditions under which a hydrogel $\mathrm{CHO}$ might be used. Although this will involve a large number of permutations of characteristics, we note in particular, that scenarios from which current testimonials about the use of hydrogels have emanated have not been investigated. These include use by elite athletes in events such as the marathon requiring high $\mathrm{CHO}$ availability from endogenous and exogenous sources to fuel prolonged exercise $(\sim 2 \mathrm{hr}+)$ of high relative and absolute intensities (Maurten, 2020; Sutehall et al., 2018). Here, the combination of issues such as high fuel requirement, high risk of gut distress, and a practical requirement for small fluid volumes intersect, making them a priority for study.

In conclusion, a small number of studies have investigated the use of commercially available $\mathrm{CHO}$ hydrogels to deliver $\mathrm{CHO}$ during exercise. So far, data do not support the claimed benefits of enhanced $\mathrm{CHO}$ delivery to the muscle, reduced GI distress, and better performance compared with the use of traditional $\mathrm{CHO}$ solutions. Future research should focus on dose and timing of hydrogel ingestion, higher exercise intensities where GI issues are more prevalent and $\mathrm{CHO}$ absorption more greatly impaired, and further mechanistic insights around endogenous $\mathrm{CHO}$ responses. Athletes may continue to use $\mathrm{CHO}$ hydrogels to meet current guidelines for endurance nutrition practices if this is their preference. While no disadvantages around the use of these specialized sports products appear to be present, based on current evidence, they do not confer metabolic or performance advantages over typical $\mathrm{CHO}$ ingestion strategies. 


\section{Acknowledgments}

All authors contributed to the preparation of this manuscript. The authors declare no conflicts of interest in the preparation of this review.

\section{References}

Achten, J., Venables, M.C., \& Jeukendrup, A.E. (2003). Fat oxidation rates are higher during running compared with cycling over a wide range of intensities. Metabolism, 52(6), 747-752. PubMed ID: 12800102 doi:10.1016/S0026-0495(03)00068-4

Barber, J.F.P., Thomas, J., Narang, B., Hengist, A., Betts, J.A., Wallis, G.A., \& Gonzalez, J.T. (2020). Pectin-alginate does not further enhance exogenous carbohydrate oxidation in running. Medicine \& Science in Sports \& Exercise, 52(6), 1376-1384. PubMed ID: 31977640 doi:10.1249/MSS.0000000000002262

Baur, D.A., Toney, H.R., Saunders, M.J., Baur, K.G., Luden, N.D., \& Womack, C.J. (2019). Carbohydrate hydrogel beverage provides no additional cycling performance benefit versus carbohydrate alone. European Journal of Applied Physiology, 119(11-12), 2599-2608. PubMed ID: 31598781 doi:10.1007/s00421-019-04240-4

Burke, L.M., Hawley, J.A., Wong, S.H., \& Jeukendrup, A.E. (2011). Carbohydrates for training and competition. Journal of Sports Sciences, 29(Suppl. 1), S17-S27. doi:10.1080/02640414.2011.585473

Burke, L.M., Jeukendrup, A.E., Jones, A.M., \& Mooses, M. (2019). Contemporary nutrition strategies to optimize performance in distance runners and race walkers. International Journal of Sport Nutrition and Exercise Metabolism, 29(2), 117-129. PubMed ID: 30747558 doi:10.1123/ijsnem.2019-0004

Burke, L.M., \& Maughan, R.J. (2015). The Governor has a sweet toothMouth sensing of nutrients to enhance sports performance. European Journal of Sport Science, 15(1), 29-40. PubMed ID: 25345670 doi:10.1080/17461391.2014.971880

Caesar, E. (2019). Inside the race to break the two-hour marathon. Retrieved from https://www.newyorker.com/sports/sporting-scene/ inside-the-race-to-break-the-two-hour-marathon-eliud-kipchoge

Cox, G.R., Clark, S.A., Cox, A.J., Halson, S.L., Hargreaves, M., Hawley, J.A., .. Burke, L.M. (2010). Daily training with high carbohydrate availability increases exogenous carbohydrate oxidation during endurance cycling. Journal of Applied Physiology, 109(1), 126134. doi:10.1152/japplphysiol.00950.2009

Coyle, E.F., Coggan, A.R., Hemmert, M.K., \& Ivy, J.L. (1986). Muscle glycogen utilization during prolonged strenuous exercise when fed carbohydrate. Journal of Applied Physiology, 61(1), 165-172. PubMed ID: 3525502 doi:10.1152/jappl.1986.61.1.165.

de Oliveira, E.P., \& Burini, R.C. (2009). The impact of physical exercise on the gastrointestinal tract. Current Opinion in Clinical Nutrition \& Metabolic Care, 12(5), 533-538. doi:10.1097/MCO. 0b013e32832e6776

de Oliveira, E.P., \& Burini, R.C. (2014). Carbohydrate-dependent, exercise-induced gastrointestinal distress. Nutrients, 6(10), 41914199. doi:10.3390/nu6104191

Flood, T.R., Montanari, S., Wicks, M., Blanchard, J., Sharpe, H., Taylor, L., ... Lee, B.J. (2020). Addition of pectin-alginate to a carbohydrate beverage does not maintain gastrointestinal barrier function during exercise in hot-humid conditions better than carbohydrate ingestion alone. Applied Physiology, Nutrition and Metabolism. Advance online publication. doi:10.1139/apnm-2020-0118

Gonzalez, J.T., \& Betts, J.A. (2019). Dietary sugars, exercise and hepatic carbohydrate metabolism. Proceedings of the Nutrition
Society, 78(2), 246-256. PubMed ID: 30348238 doi:10.1017/ S0029665118002604

Gonzalez, J.T., Fuchs, C.J., Smith, F.E., Thelwall, P.E., Taylor, R., Stevenson, E.J., ... van Loon, L.J.C. (2015). Ingestion of glucose or sucrose prevents liver but not muscle glycogen depletion during prolonged endurance-type exercise in trained cyclists. American Journal of Physiology: Endocrinology and Metabolism, 309(12), E1032-E1039. PubMed ID: 26487008 doi:10.1152/ajpendo.00376. 2015

Hawley, J.A., Bosch, A.N., Weltan, S., Dennis, S., \& Noakes, T. (1994). Glucose kinetics during prolonged exercise in euglycaemic and hyperglycaemic subjects. Pflügers Archiv: European Journal of Physiology, 426(5), 378-386. PubMed ID: 8015888 doi:10.1007/ BF00388300.

Ioannidis, J.P., \& Trikalinos, T.A. (2007). The appropriateness of asymmetry tests for publication bias in meta-analyses: A large survey. Canadian Medical Association Journal, 176(8), 1091-1096. PubMed ID: 17420491 doi:10.1503/cmaj.060410

Jeukendrup, A.E. (2010). Carbohydrate and exercise performance: the role of multiple transportable carbohydrates. Current Opinion in Clinical Nutrition \& Metabolic Care, 13(4), 452-457. PubMed ID: 20574242 doi:10.1097/MCO.0b013e328339de9f

Jeukendrup, A.E. (2017). Training the gut for athletes. Sports Medicine, 47(Suppl. 1), 101-110. PubMed ID: 28332114 doi:10.1007/s40279017-0690-6

Jeukendrup, A.E., Wagenmakers, A.J., Stegen, J., Gijsen, A.P., Brouns, F., \& Saris, W. (1999). Carbohydrate ingestion can completely suppress endogenous glucose production during exercise. American Journal of Physiology: Endocrinology and Metabolism, 276(4), E672-E683. doi:10.1152/ajpendo.1999.276.4.E672.

King, A.J., O’Hara, J.P., Arjomandkhah, N.C., Rowe, J., Morrison, D.J., Preston, T., \& King, R.F.G.J. (2019). Liver and muscle glycogen oxidation and performance with dose variation of glucose-fructose ingestion during prolonged $(3 \mathrm{~h})$ exercise. European Journal of Applied Physiology, 119(5), 1157-1169. PubMed ID: 30840136 doi:10.1007/s00421-019-04106-9

King, A.J., O’Hara, J.P., Morrison, D.J., Preston, T., \& King, R. (2018). Carbohydrate dose influences liver and muscle glycogen oxidation and performance during prolonged exercise. Physiological Reports, 6(1), e13555. doi:10.14814/phy2.13555

Losnegard, T., Schafer, D., \& Hallen, J. (2014). Exercise economy in skiing and running. Frontiers in Physiology, 5(5), 5. PubMed ID: 24478718 doi: $10.3389 /$ fphys.2014.00005

Marciani, L., Lopez-Sanchez, P., Pettersson, S., Hoad, C., Abrehart, N., Ahnoff, M., \& Ström, A. (2019). Alginate and HM-pectin in sportsdrink give rise to intra-gastric gelation in vivo. Food \& Function, 10(12), 7892-7899. PubMed ID: 31793602 doi:10.1039/ C9FO01617A

Margolis, L.M., Wilson, M.A., Whitney, C.C., Carrigan, C.T., Murphy, N.E., Hatch, A.M., ... Pasiakos, S.M. (2019). Exercising with low muscle glycogen content increases fat oxidation and decreases endogenous, but not exogenous carbohydrate oxidation. Metabolism, 97, 1-8. PubMed ID: 31095946 doi:10.1016/j.metabol.2019.05.003

Maurten. (2020). The world's fastest sports fuel. Retrieved from https:// www.maurten.com/achievements

McCubbin, A.J., Zhu, A., Gaskell, S.K., \& Costa, R.J.S. (2019). Hydrogel carbohydrate-electrolyte beverage does not improve glucose availability, substrate oxidation, gastrointestinal symptoms or exercise performance, compared with a concentration and nutrient-matched placebo. International Journal of Sport Nutrition and Exercise Metabolism,30(1), 25-33. doi:10.1123/ijsnem.2019-0090 
Mears, S.A., Boxer, B., Sheldon, D., Wardley, H., Tarnowski, C.A., James, L.J., \& Hulston, C.J. (2020a). Sports drink intake pattern affects exogenous carbohydrate oxidation during running. Medicine \& Science in Sports \& Exercise. Advance online publication. doi:10. 1249/MSS.0000000000002334

Mears, S.A., Worley, J., Mason, G.S., Hulston, C.J., \& James, L.J. (2020b). Addition of sodium alginate and pectin to a carbohydrate-electrolyte solution does not influence substrate oxidation, GI comfort or cycling performance. Applied Physiology, Nutrition and Metabolism, 45(6), 675-678. doi:10.1139/apnm-2019-0802

Menzies, C., Wood, M., Thomas, J., Hengist, A., Walhin, J.P., Jones, R., . . . Betts, J.A. (2020). Frequent carbohydrate ingestion reduces muscle glycogen depletion and postpones fatigue relative to a single bolus. International Journal of Sport Nutrition and Exercise Metabolism. 30(1), 25-33. doi:10.1123/ijsnem.2019-0291

Newell, M.L., Wallis, G.A., \& Galloway, S.D.R. (2014). Impact of CHO nutrition on exercise metabolism and performance. Agro FOOD Industry Hi-Tech, 25(2), 32-36.

O'Brien, W.J., Stannard, S.R., Clarke, J.A., \& Rowlands, D.S. (2013). Fructose-maltodextrin ratio governs exogenous and other $\mathrm{CHO}$ oxidation and performance. Medicine \& Science in Sports \& Exercise, 45(9), 1814-1824. PubMed ID: 23949097 doi:10.1249/MSS. 0b013e31828e12d4

Pettersson, S., Edin, F., Bakkman, L., \& McGawley, K. (2019). Effects of supplementing with an $18 \%$ carbohydrate-hydrogel drink versus a placebo during whole-body exercise in $-5{ }^{\circ} \mathrm{C}$ with elite cross-country ski athletes: A crossover study. Journal of the International Society of Sports Nutrition, 16(1), 46. PubMed ID: 31655603 doi:10.1186/ s12970-019-0317-4

Rehrer, N.J., Wagenmakers, A.J., Beckers, E.J., Hall, D., Leiper, J.B., Brouns, F., ... Saris, W.H. (1992). Gastric emptying, absorption, and carbohydrate oxidation during prolonged exercise. Journal of Applied Physiology, 72(2), 468-475. PubMed ID: 1559921 doi:10. 1152/jappl.1992.72.2.468.

Robert, J.J., Koziet, J., Chauvet, D., Darman, D., Desjeux, J.F., \& Young, V.R. (1987). Use of 13C-labeled glucose for estimating glucose oxidation: Some design considerations. Journal of Applied Physiology, 63(5), 1725-1732. PubMed ID: 3121572 doi:10.1152/jappl. 1987.63.5.1725.

Rowlands, D.S., Houltham, S., Musa-Veloso, K., Brown, F., Paulionis, L., \& Bailey, D. (2015). Fructose-glucose composite carbohydrates and endurance performance: Critical review and future perspectives. Sports Medicine, 45(11), 1561-1576. PubMed ID: 26373645 doi:10. 1007/s40279-015-0381-0

Shi, X., Summers, R.W., Schedl, H.P., Flanagan, W., Chang, R., \& Gisolfi, C.V. (1995). Effects of carbohydrate type and concentration and solution osmolality on water absorption. Medicine \& Science in Sports \& Exercise, 27(12), 1607-1615. PubMed ID: 8614315 doi:10.1249/00005768-199512000-00005.
Smith, J.W., Zachwieja, J.J., Peronnet, F., Passe, D.H., Massicotte, D., Lavoie, C., \& Pascoe, D.D. (2010). Fuel selection and cycling endurance performance with ingestion of [13C]glucose: Evidence for a carbohydrate dose response. Journal of Applied Physiology, 108(6), 1520-1529. PubMed ID: 20299609 doi:10.1152/japplphysiol. 91394.2008

Snipe, R.M.J., Khoo, A., Kitic, C.M., Gibson, P.R., \& Costa, R.J.S. (2017). Carbohydrate and protein intake during exertional heat stress ameliorates intestinal epithelial injury and small intestine permeability. Applied Physiology, Nutrition and Metabolism, 42(12), 1283-1292. PubMed ID: 28777927 doi:10.1139/apnm2017-0361

Stellingwerff, T., \& Cox, G.R. (2014). Systematic review: Carbohydrate supplementation on exercise performance or capacity of varying durations. Applied Physiology, Nutrition and Metabolism, 39(9), 998-1011. PubMed ID: 24951297 doi:10.1139/apnm-20140027

Sutehall, S., Galloway, S.D.R., Bosch, A., \& Pitsiladis, Y. (2020). Addition of an alginate hydrogel to a carbohydrate beverage enhances gastric emptying. Medicine \& Science in Sports \& Exercise. Advance online publication. doi:10.1249/MSS.0000000000002301

Sutehall, S., Muniz-Pardos, B., Bosch, A., Di Gianfrancesco, A., \& Pitsiladis, Y. (2018). Sports drinks on the edge of a new era. Current Sports Medicine Reports, 17(4), 112-116. PubMed ID: 29629968 doi:10.1249/JSR.0000000000000475.

ter Steege, R.W., \& Kolkman, J.J. (2012). Review article: The pathophysiology and management of gastrointestinal symptoms during physical exercise, and the role of splanchnic blood flow. Alimentary Pharmacology \& Therapeutics, 35(5), 516-528. doi:10.1111/j.1365-2036. 2011.04980.x

Thomas, D.T., Erdman, K.A., \& Burke, L.M. (2016). American College of Sports Medicine Joint Position Statement. Nutrition and athletic performance. Medicine \& Science in Sports \& Exercise, 48(3), 543-568. PubMed ID: 26891166 doi:10.1249/MSS.0000000000000852

Tsintzas, K., Williams, C., Boobis, L., \& Greenhaff, P. (1995). Carbohydrate ingestion and glycogen utilization in different muscle fibre types in man. Journal of Physiology, 489(1), 243-250. doi:10.1113/ jphysiol.1995.sp021046.

Tsintzas, K., Williams, C., Boobis, L., \& Greenhaff, P. (1996). CHO ingestion and single muscle fibre glycogen metabolism during prolonged running in men. Journal of Applied Physiology, 81(2), 801-809. PubMed ID: 8872649 doi:10.1152/jappl.1996. 81.2.801.

Wallis, G.A., Dawson, R., Achten, J., Webber, J., \& Jeukendrup, A.E. (2006). Metabolic response to carbohydrate ingestion during exercise in males and females. American Journal of Physiology: Endocrinology and Metabolism, 290(4), E708-E715. PubMed ID: 16278245 doi:10.1152/ajpendo.00357.2005 